\title{
Estratégia para amostragem da população e da paisagem em pesquisas sobre uso e cobertura da terra*
}

\author{
Álvaro de Oliveira D’Antona** \\ Leah Karin VanWey ${ }^{\star \star \star}$
}

\begin{abstract}
Este artigo apresenta a estratégia usada em uma pesquisa realizada em Santarém - PA, em 2003, para amostrar lotes delimitados em mapas do Incra e as unidades domésticas ali existentes, de modo a capturar as mudanças populacionais e ambientais na escala das propriedades rurais e na da região. A amostra foi selecionada conforme a distribuição dos lotes ao longo de quatro eixos rodoviários relacionados a distintos processos de ocupação. Em cada um dos eixos foram selecionadas aleatoriamente 20 células com $9 \mathrm{~km}^{2}$. Em cada uma delas, foram selecionados aleatoriamente os lotes que deveriam ser visitados. No campo, entrevistaram-se todas as unidades domésticas existentes em cada um dos lotes selecionados. Descreve-se a amostragem por segmentação sucessiva da área de estudo, destacando-se como os dados resultantes podem ser usados diferentemente nas escalas das unidades domésticas, dos lotes e da região, conforme as questões e variáveis de interesse.
\end{abstract}

Palavras-chave: Amazônia brasileira. Uso da terra. Unidade doméstica. Amostragem em estágios múltiplos.

\section{Introdução}

O desmatamento na Amazônia, entre 1978 e 2004, estimado em aproximadamente 528 mil quilômetros quadrados (INPE, 2006), tem implicações negativas do ponto de vista local, regional e global. Escassez de recursos naturais abundantes no passado, perda de biodiversidade, emissão de carbono na atmosfera e efeitos sobre o aquecimento global são aspectos recorrentemente discutidos nos meios acadêmicos e não-acadêmicos.

$\mathrm{Na}$ busca por uma compreensão extensiva e em múltiplos níveis do processo de desmatamento da Amazônia, as suas genericamente denominadas "dimensões humanas" têm merecido crescente atenção, mesmo em projetos de larga escala

\footnotetext{
* Versão revista e ampliada de texto originalmente apresentado no XIV Encontro Nacional de Estudos Populacionais, Abep, realizado em Caxambu/ MG - Brasil, de 20 a 24 de setembro de 2006.

Os autores agradecem ao National Institute for Child Health and Human Development pelo suporte financeiro ao projeto de pesquisa (HD35811); aos membros do ACT/Indiana University e aos pesquisadores de Santarém, por sua assistência; ao Escritório Santarém do Large-Scale Biosphere Atmosphere experiment in Amazonia, pelo apoio logístico durante o trabalho de campo; e aos residentes na área de estudo, por sua colaboração e pelo tempo dedicado.

** Bacharel em Ciências Econômicas (IE/Unicamp), mestre em Antropologia (IFCH/Unicamp), doutor em Ciências Sociais (IFCH/Unicamp). Pesquisador do Núcleo de Estudos de População, Universidade Estadual de Campinas (Nepo/ Unicamp). Associado ao Anthropological Center for Research and Training on Global Environmental Change, Indiana University (ACT/IU).

${ }^{\star \star \star}$ Mestre e doutora em Sociologia (University of North Carolina). Professora assistente de Sociologia do Departamento de Sociologia da Indiana University (IU). Associada ao ACT/IU e ao Center for the Study of Institutions, Population and Environmental Change (CIPEC/IU).
} 
(BATISTELLA; MORAN, 2005). Os estudos voltados para o modo de vida dos produtores rurais têm se destacado pela abordagem multidisciplinar que adotam (LIVERMAN et al., 1998) e por oferecerem explicações na escala em que as mudanças no uso da terra realmente ocorrem, ou seja, na escala dos estabelecimentos rurais (EVANS; MORAN, 2002).

Pesquisas realizadas nesta escala micro, das unidades domésticas e dos lotes de terra, têm mostrado que estabelecimentos agropecuários são unidades territoriais essenciais para conectar população e ambiente, permitindo corresponder reciprocamente variáveis socioeconômicas e demográficas com dados biofísicos. No entanto, para que os resultados desse tipo de estudo sejam extrapolados para áreas maiores (por exemplo, um projeto de assentamento, um município, ou região), é necessário que se definam estratégias de amostragem que permitam generalizações sobre a população e a paisagem, a partir de dados de campo (surveys ou etnografias) coletados em um limitado número de famílias.

Neste texto discute-se a estratégia de amostragem em estágios múltiplos, utilizada em survey socioconômico e demográfico, realizado em 2003, em área de estudo localizada entre os municípios de Santarém e Belterra, no Pará. A estratégia de amostragem baseada na distribuição espacial dos lotes rurais, definidos em mapas do Instituto Nacional de Colonização e Reforma Agrária (Incra), e o desenho do survey permitem que os dados de indivíduos, unidades domésticas e lotes sejam representativos e espacialmente relacionados com caracteres biofísicos.

Na primeira parte deste artigo, contextualiza-se o tipo de pesquisa e localiza-se a área de estudo. Na segunda, a estratégia de amostragem e os instrumentos do survey são apresentados. A terceira parte traz os resultados do trabalho de campo e a parte final contém os prós e contras da estratégia empregada.

Ainda que a metodologia tenha sido aplicada a um estudo voltado para mudanças no uso e na cobertura da terra, a estratégia adotada é apropriada a qualquer aspecto da paisagem - base do meio físico que precise ser generalizado, incluindo, por exemplo, solos, produção e áreas plantadas. Trata-se, portanto, de relevante estratégia para estudos de população e ambiente.

\section{Contextualização}

Existe vasta bibliografia relacionando componentes socioeconômicos e demográficos às mudanças no uso e cobertura da terra em várias escalas e contextos. Especialmente sobre a Amazônia brasileira e na escala das propriedades rurais, trabalhos em áreas de colonização (WOOD; SCHMINK, 1979; MORAN, 1981; FEARNSIDE, 1986; SCHMINK; WOOD, 1992) enfocam vários aspectos das dimensões humanas das modificações no uso da terra, tais como a percepção ambiental e as estratégias de uso da terra (PICHÓN, 1997; MUCHAGATA; BROWN, 2000; MORAN et al., 2002; BROWDERI et al., 2004) e a demografia das unidades domésticas e as trajetórias de uso da terra (PICHÓN, 1997; MARQUETTE, 1998; MCCRACKEN et al., 1999; BRONDÍZIO et al., 2002; MCCRACKEN et al., 2002; WALKER et al., 2002; SIQUEIRA et al., 2003).

Os trabalhos na escala micro normalmente partem de uma base de dados georreferenciados que incluem o mapa de lotes rurais, uma coleção de imagens de satélite com dados sobre cobertura da terra e outras fontes de dados secundários, como mapa de solos e dados censitários (BRONDÍZIO et al., 1996; MCCRACKEN et al., 1999; BRONDÍZIO et al., 2002; MCCRACKEN et al., 2002). Sobre tal base, organiza-se o trabalho de campo (geralmente survey acompanhado de outros instrumentos) para coleta de informações agregadas por unidade doméstica e lote de terra. Nos trabalhos de campo, são reunidos dados socioeconômicos, demográficos e de uso e cobertura da terra nas unidades selecionadas na amostra. As informações tabuladas são incluídas na base de dados, permitindo que características das unidades domésticas e dos lotes sejam cruzadas com outras fontes de dados.

A capacidade explicativa e a possibilidade de generalização dos resultados 
obtidos na escala micro dependem da adequada conexão entre dados das unidades domésticas (composição da unidade doméstica, por exemplo), informações dos estabelecimentos agropecuários (história de uso da terra, por exemplo) e dados biofísicos (tipo de solo, disponibilidade de água, entre outros). Isso torna os lotes, e não apenas as unidades domésticas, essenciais unidades de amostragem. Conseqüentemente, a estratégia de amostragem precisa capturar, no espaço, a variabilidade de situações sociais, fundiárias e biofísicas existentes dentro da área de estudo e, adicionalmente, não deve impedir nem dificultar a coleta de dados no campo, sobretudo no que diz respeito à localização das unidades domésticas a serem entrevistadas.

Por permitir maior controle da seleção da amostra e facilitar a locomoção durante o trabalho de campo, a segmentação sucessiva da área de estudo foi a estratégia adotada para a amostragem de lotes para o levantamento socioeconômico e demográfico (survey) realizado pelo projeto Amazonian Deforestation and the Structure of Households, entre junho e setembro de 2003, em Santarém, Pará. ${ }^{1}$

\section{A amostragem na área de estudo}

Com pouco mais de $1.800 \mathrm{~km}^{2}$, a área de estudo (Figura 1) ocupa parte dos municípios de Santarém e Belterra, no Pará, estando predominantemente na zona rural do primeiro.

O município de Santarém possui uma área de $24.314,4 \mathrm{~km}^{2}$, com população de 262.538 habitantes, sendo $71 \%$ urbana (IBGE, 2000). Na confluência dos Rios Tapajós e Amazonas, a sede municipal de Santarém é um antigo centro urbano, cuja existência remonta a ocupação colonial através do Rio Amazonas (REIS, 1979; GUILHON, 1983). Trata-se, ainda hoje, de importante porto entre Manaus-AM e

FIGURA 1

Área de estudo nos municípios de Santarém e Belterra

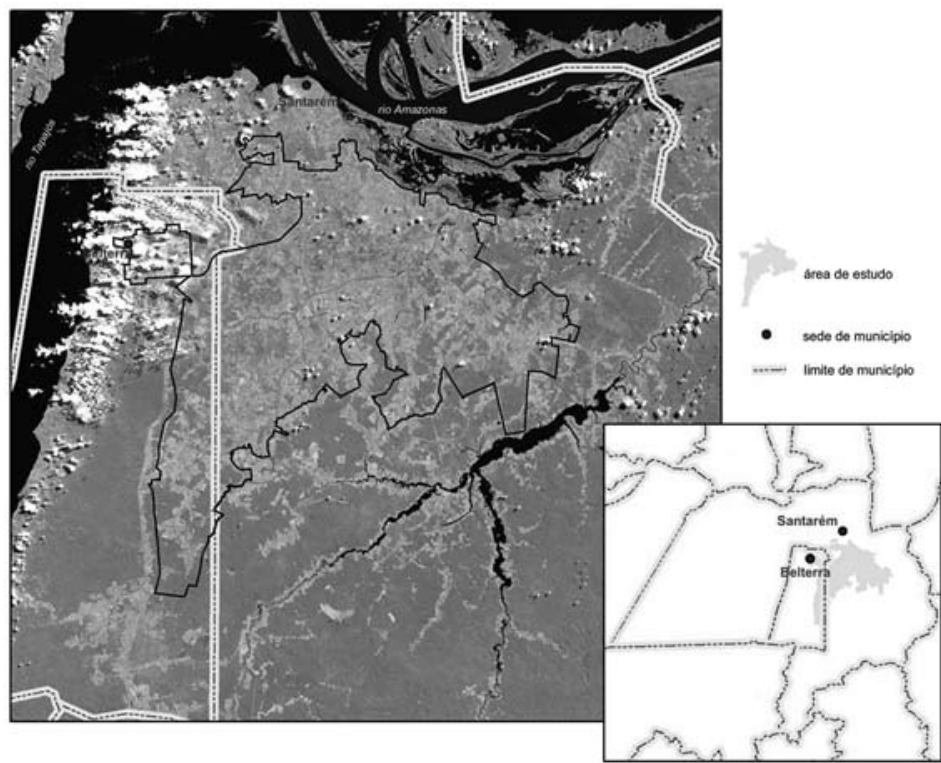

Fonte: Elaboração dos autores, a partir do acervo do ACT/Indiana University.

\footnotetext{
1 Projeto do Anthropological Center for Research and Training on Global Environmental Change, da Indiana University (ACT/IU), em parceria com o Núcleo de Estudos de População, da Universidade Estadual de Campinas (Nepo/Unicamp), realizado com o apoio do National Institute for Child Health and Human Development (HD35811).
} 
Belém-PA, sobretudo pela inauguração, em 2003, de terminal da empresa Cargill para o embarque de soja e outros grãos (STEWARD, 2004, p. 2). Com área de $2.628,9 \mathrm{~km}^{2}$ e população de 14.594 habitantes, 35\% urbana (IBGE, 2000), o município de Belterra está incrustado no município de Santarém, do qual se emancipou em 1995. Grande parte de sua área é ocupada pela Floresta Nacional do Tapajós, sendo sua sede municipal baseada em agrovila criada pela Companhia Ford, nas primeiras décadas do século $X X$, para abrigar os empregados em seu projeto de cultivo de seringueiras (REIS, 1979; GUILHON, 1983).

No último século a região de Santarém passou por vários ciclos econômicos. O mais recente deles, em curso, deve-se à introdução do cultivo mecanizado de soja e à chegada de produtores do centro-oeste, o que afetou o mercado fundiário - tanto pelo aumento do preço da terra, quanto pela concentração fundiária - e a mobilidade populacional - o êxodo de algumas partes do município causou inclusive o desapare- cimento de vilas, convertidas em áreas de agricultura mecanizada.

Com o objetivo de entender as mudanças no uso da terra e suas relações com componentes socioeconômicos e demográficos, foi realizado o survey em 2003. Para a escolha da área de estudo, pesaram o fato de se tratar de área de ocupação antiga - o que permite investigar modificações intergeracionais no uso da terra - e a possibilidade de comparação com porções colonizadas mais recentemente, como, por exemplo, área de projeto de colonização ao longo da Rodovia Transamazônica, entre os municípios de Altamira e Uruará, no Pará. ${ }^{2}$

\section{Estratégia de amostragem}

A amostra foi selecionada levando-se em conta a distribuição espacial de lotes de terra situados em áreas de projeto de colonização e em porções com ocupação espontânea, conforme mapas do Incra.

Tendo por referência imagens do Landsat TM (1997 e 1999) e cartas topográficas do

FIGURA 2

Malha de lotes do Incra na área de estudo nos municípios de Santarém e Belterra

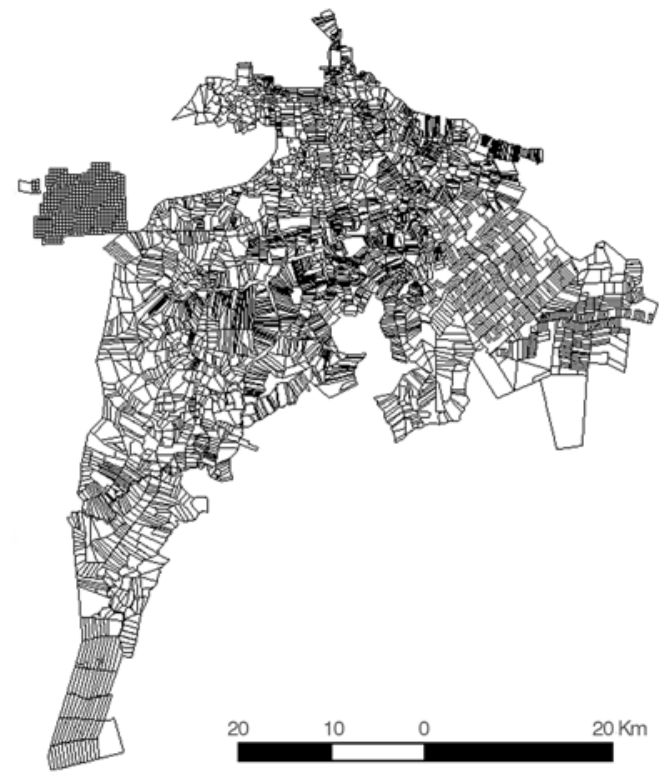

Fonte: Elaboração dos autores, a partir do acervo do ACT/Indiana University.

\footnotetext{
2 Tal área de estudo também faz parte do projeto Amazonian Deforestation and the Structure of Households, tendo sido visitada entre junho e setembro de 2005.
} 
IBGE, na escala 1:100.000, os mapas de lotes do Incra, na escala 1:50.000, foram digitalizados, georreferenciados evetorizados, dando origem a uma única malha de lotes (Figura 2).

$\mathrm{O}$ projeto de pesquisa tinha interesse em investigar os efeitos do tempo de ocupação do lote sobre as decisões de uso da terra por parte das unidades domésticas. No entanto, em se tratando de área de colonização antiga, não seria possível usar imagens de satélite para estratificar a amostra de acordo com o ano em que o lote de terra foi desmatado pela primeira vez. ${ }^{3}$ Assim, a estratificação foi feita a partir da divisão da área de estudo em quatro regiões, que refletem a história de ocupação desde 1930 (MORAN; BRONDÍZIO; VANWEY, 2005).
As quatro principais vias de acesso terrestre, incluindo a Rodovia federal Santarém-Cuiabá (BR-163), foram usadas como distintos eixos de influência e de ocupação para delimitar as regiões. Para a estratificação dentro de cada região, foi aplicada uma grade com 281 células de $3 \mathrm{~km}$ por $3 \mathrm{~km}$ sobre 5.086 lotes no mapa de propriedades do Incra (Figura 3).

Em cada uma das quatro regiões foram selecionadas aleatoriamente 20 células (Figura 4), de modo a se obter uma amostra com significativa cobertura espacial. 0 mesmo número de células foi selecionado em todas as regiões, a fim de se chegar a conclusões estatísticas sobre cada uma das quatro regiões.

FIGURA 3

Células nas quatro regiões da área de estudo

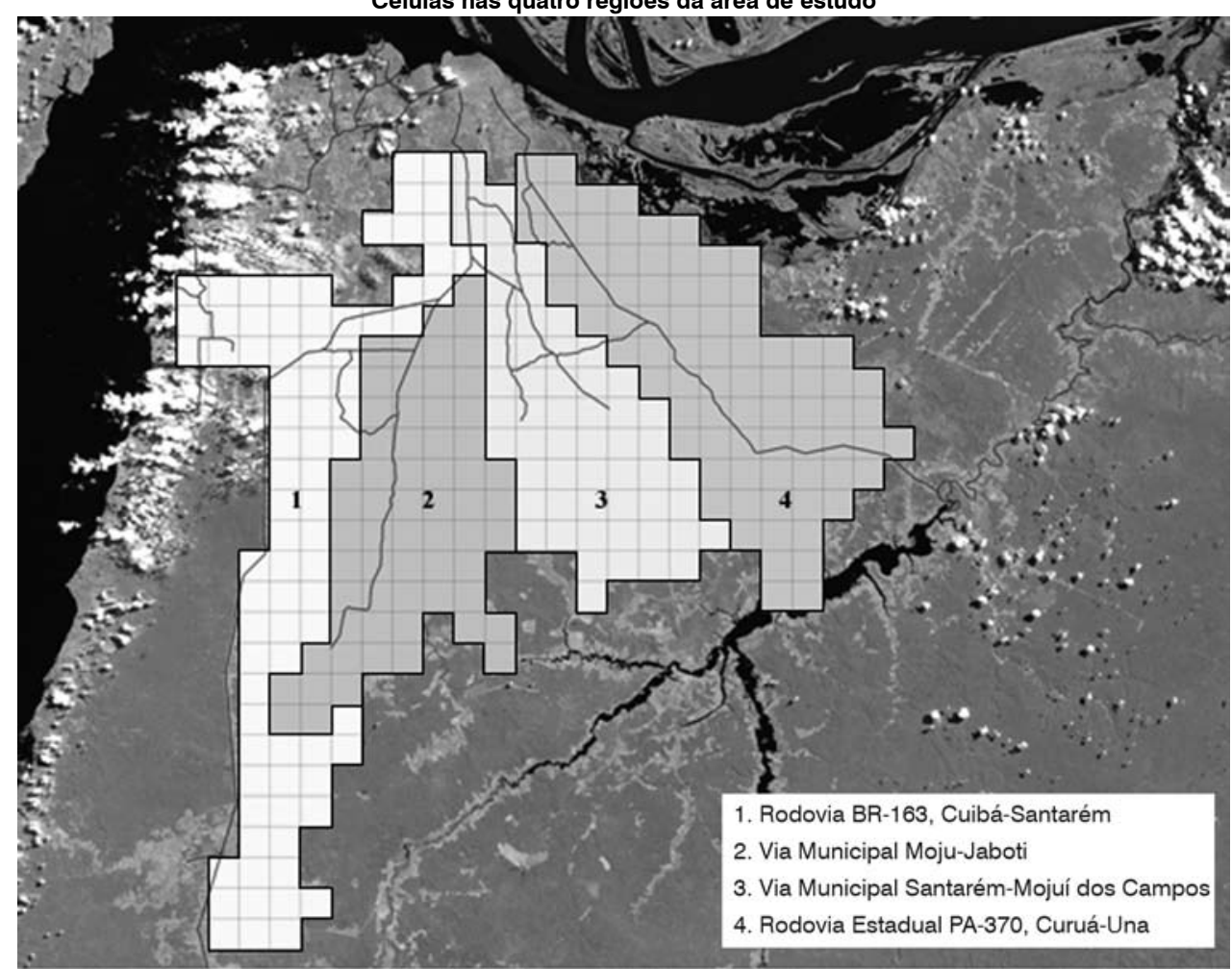

Fonte: Elaboração dos autores, a partir do acervo do ACT/Indiana University.

${ }^{3}$ O primeiro desmatamento pode ser usado como um indicativo do tempo de ocupação dos lotes de terra, permitindo que se estratifique a amostra de acordo com a "idade do lote". Tal procedimento não se aplica à área de estudo em Santarém, pois a mesma começou a ser desmatada antes da década de 70 , não havendo imagens de satélite que documentem os estágios iniciais da mudança na cobertura da terra. 
FIGURA 4

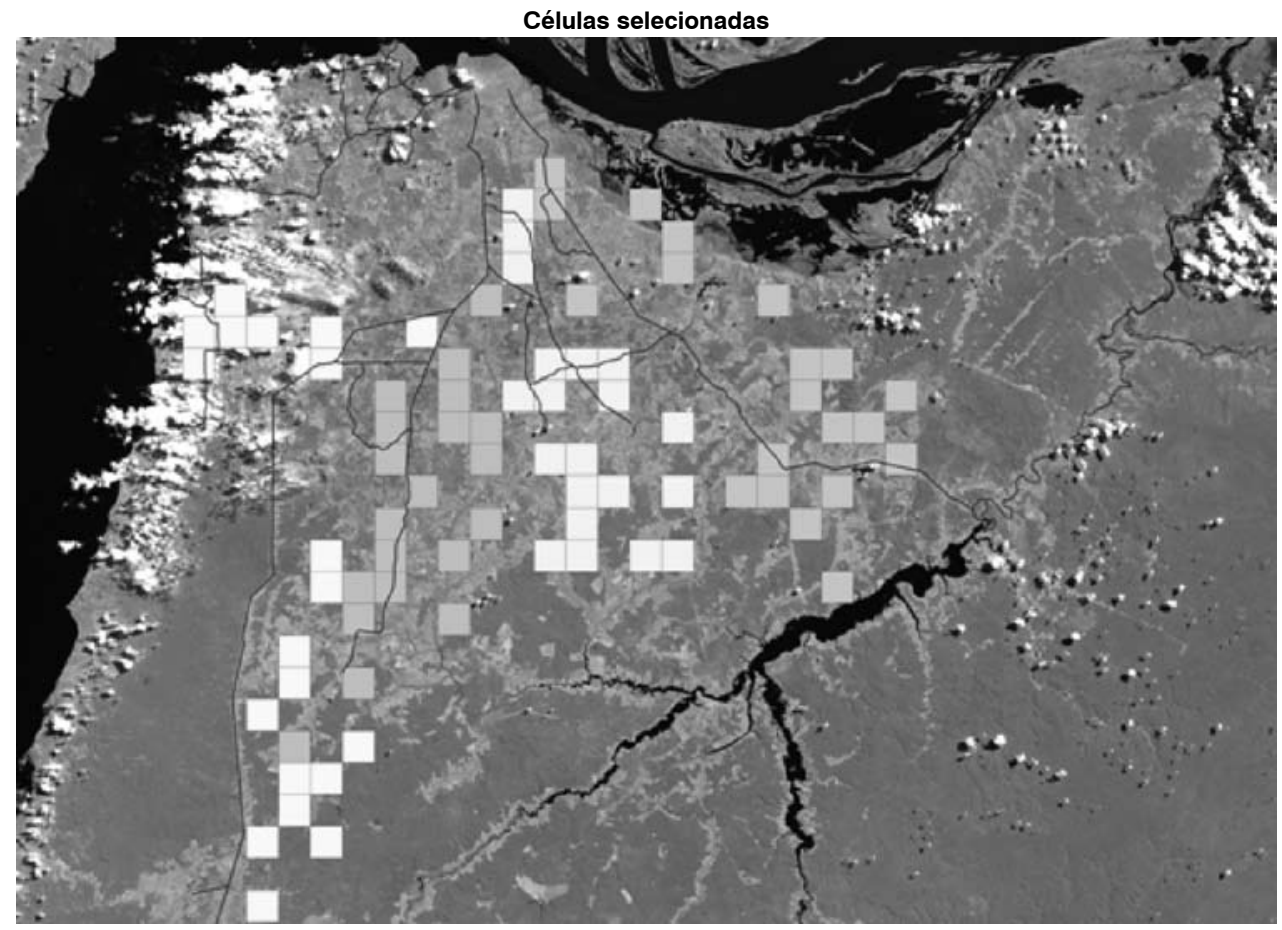

Fonte: Elaboração dos autores, a partir do acervo do ACT/Indiana University.

Nota: As células identificadas nesta figura não correspondem àquelas realmente amostradas para que se garanta o anonimato dos entrevistados.

Em cada uma das 80 células definidas, foram selecionados aleatoriamente lotes do Incra, o que facilita o acesso às propriedades durante o trabalho de campo (as propriedades estão concentradas), sem que se comprometa a distribuição das propriedades em cada região e, conseqüentemente, em toda a área de estudo. Foram usualmente selecionados nove lotes em cada célula para serem realizadas as entrevistas, tendo sido considerado pertencente à célula o lote cujo centro estivesse dentro dela. Outros cinco lotes foram selecionados em cada célula, como parte de uma amostra alternativa, para substituir aqueles lotes que eventualmente não pudessem ser entrevistados durante o trabalho de campo (por exemplo, em caso de recusa dos moradores). A Figura 5 ilustra a seleção de lotes da amostra e dos alternativos. No primeiro exemplo (célula da es- querda), todos os lotes foram selecionados para a amostra, pois eram em quantidade inferior a nove - nos casos em que a célula continha menos de 14 propriedades, foram escolhidos o maior número possível de lotes para a amostra; portanto, nem sempre eram escolhidos lotes alternativos. No segundo exemplo (direita), nove lotes foram incluídos na amostra, outros cinco foram escolhidos como alternativos e os demais ficaram fora da amostra.

O dimensionamento da grade de células e o número de unidades selecionadas levaram em consideração as características dos lotes na área de estudo, os recursos disponíveis para o trabalho de campo e a meta de ter dados sobre 550 lotes. $\mathrm{Na}$ área de estudo, os lotes têm, em média, aproximadamente 35 hectares, havendo grande variação de tamanho e formatos. ${ }^{4} \mathrm{~A}$

\footnotetext{
${ }^{4}$ Ao contrário do que ocorre em áreas exclusivamente ocupadas por projetos de colonização, nos quais os lotes têm tamanho similar, são normalmente retangulares e uniformemente distribuidos ao longo de malha viária com estradas perpendiculares, na área de estudo em Santarém não há organização/distribuição regular dos lotes, como se pode observar na Figura 2.
} 
FIGURA 5

llustração de lotes selecionados nas células
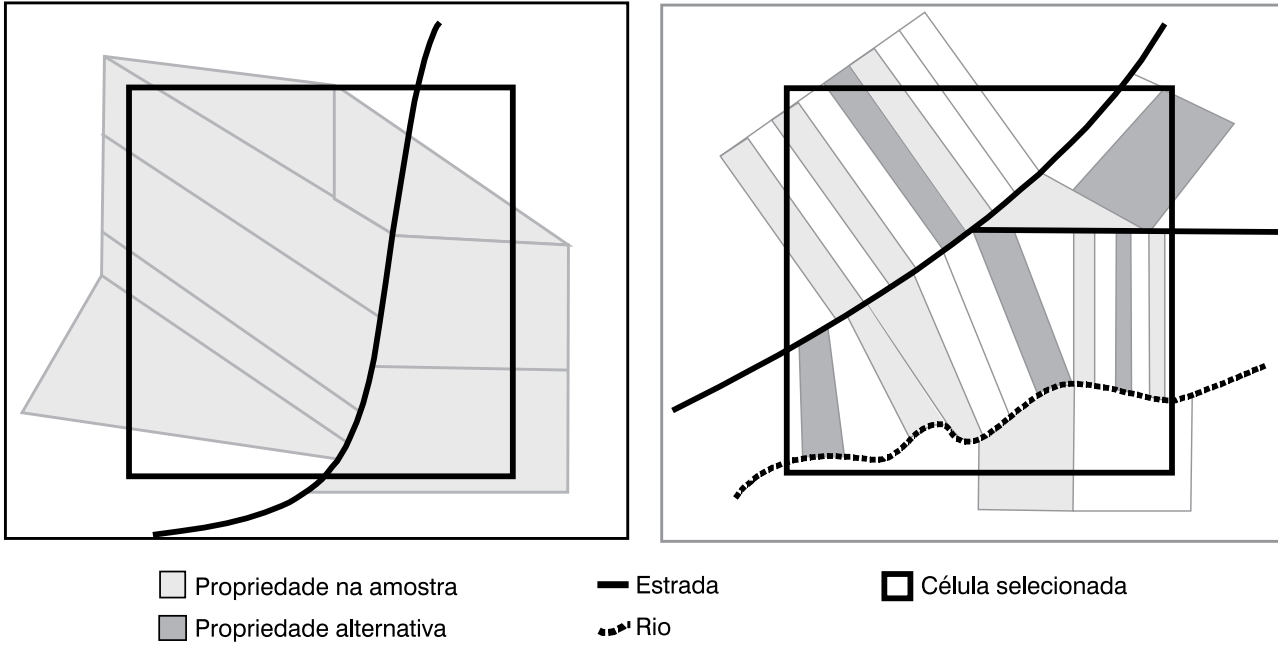

$\square$ Célula selecionada

Fonte: Elaboração dos autores, a partir do acervo do ACT/Indiana University.

Nota: As figuras não correspondem a células nem a lotes reais para garantir o anonimato dos entrevistados.

distribuição irregular dos lotes e as difíceis condições de acesso (além do próprio tamanho da área de estudo) impuseram a busca por meios de se obter a informação com representatividade do contexto espacial e demográfico, reduzindo custos de deslocamento e tempo de permanência no campo. Testes feitos com células de tamanhos distintos mostraram que o padrão $3 \mathrm{~km} \times 3 \mathrm{~km}$ - que agrega em média 18 lotes - seria o ideal, dadas as características dos lotes e os objetivos do trabalho. Em células menores que $3 \mathrm{~km} \times 3 \mathrm{~km}$, o número de lotes seria reduzido e haveria a necessidade de se selecionarem mais células, o que dificultaria o trabalho de campo. Em células maiores que $3 \mathrm{~km} \times 3 \mathrm{~km}$, agregar-se-ia um número maior de lotes, o que, embora pudesse facilitar o trabalho de campo, comprometeria a representatividade espacial da amostra, posto que a seleção de um número muito pequeno de células não proporcionaria uma boa cobertura das características físicas em toda a área de estudo.

\section{Instrumentos da pesquisa}

Os instrumentos de pesquisa foram desenhados para a coleta de dados de unidades domésticas existentes em propriedades agropecuárias familiares. ${ }^{5}$ Nos questionários foram reunidas perguntas sobre características da unidade doméstica (econômicas e demográficas) e da propriedade (produção, uso e cobertura da terra) no presente e no passado, o que permite estabelecer paralelos entre a história das unidades domésticas e a das propriedades. Para localizar as unidades domésticas e registrar as transformações no uso e cobertura da terra ao longo do tempo, foram criados mapas baseados em imagens de satélite e formulário para o desenho dos limites das propriedades e para a localização de usos da terra, casas e outras edificações existentes.

Foram definidos procedimentos para captar a fragmentação de lotes decorrente de partilha entre herdeiros ou mesmo venda,

\footnotetext{
5 O termo "propriedade" refere-se aos estabelecimentos agropecuários visitados no trabalho de campo, enquanto "lote" é usado em referência aos limites dos estabelecimentos amostrados. A distinção é importante para destacar que a propriedade encontrada em 2003 no local do lote amostrado poderia ser igual, menor ou maior do que o lote mapeado pelo Incra.
} 
bem como a agregação de lotes. Contudo, o desenho original dos instrumentos assumia que os novos donos também seriam pequenos produtores, como os originais.

Planejou-se entrevistar todas as unidades domésticas existentes e, assim, essa amostra tem um nível a mais de estratificação do que a de lotes, tornando-se representativa de todas as unidades domésticas na região e não somente daquelas de proprietários de estabelecimentos agropecuários.

\section{Resultados}

Uma vez no campo, duplas de entrevistadores (um homem e uma mulher) visitaram os lotes previamente selecionados. Ao entrevistador coube o contato com o homem "dono do lote" (ou "chefe da unidade doméstica") para aplicação de questionários sobre características físicas da propriedade e produção agropecuária. À entrevistadora coube o contato com a mulher "dona da casa" (ou mulher do "chefe da unidade doméstica") para aplicação de questionário sobre composição e características da unidade doméstica, contracepção e história reprodutiva - os dois últimos temas também abordados com todas as outras mulheres maiores de 15 anos residentes nas casas visitadas.

Em cada lote da amostra, questionários foram aplicados a todas as unidades domésticas existentes, mesmo nas situações em que os limites da propriedade encontrada não correspondiam aos do lote original. Quando mais de uma propriedade era encontrada no lugar de um lote do Incra (por exemplo, quando o lote foi dividido entre herdeiros), procurou-se aplicar questionários em todas as unidades domésticas de todas as propriedades existentes na área do lote original, de modo que se obtivesse informação sobre a sua fragmentação. Quando o lote original foi agregado a outros lotes, formando uma propriedade maior, procurou-se aplicar questionários a todas as unidades domésticas existentes na propriedade encontrada.

A experiência do campo revelou uma estrutura fundiária diferente da esperada, o que muitas vezes impossibilitou a aplicação de questionários. Alguns lotes originais foram subdivididos em várias propriedades ao longo dos anos - algumas delas dificilmente podem ser denominadas de estabelecimentos agropecuários, dadas as suas dimensões (em torno de $250 \mathrm{~m}^{2}$, semelhante a lotes urbanos), localização (em vilas/comunidades) e uso (servem apenas como local de moradia). Outros lotes do Incra foram agregados em grandes estabelecimentos não-familiares (alguns deles com mais de 50 lotes originais), geralmente destinados à pecuária $\mathrm{e}$, mais recentemente, ao plantio mecanizado de arroz e soja.

No trabalho de campo foram identificadas 460 propriedades sobre 587 lotes do Incra que fazem parte da amostra. Conforme a rotina de campo mencionada anteriormente, foram aplicados questionários em 243 das 460 propriedades, que são predominantemente estabelecimentos agropecuários familiares, onde existem 401 unidades domésticas e residem 1.849 indivíduos.

Nas outras 217 propriedades identificadas não foram realizadas entrevistas por pelo menos um dos seguintes motivos: não tinham uso agropecuário; estavam desocupadas (sem residentes); estavam abandonadas (sem uso) ou faziam parte de grandes propriedades (pastagens e áreas mecanizadas geralmente sem moradores). Em tais casos, características gerais dos eventuais ocupantes (como origem e número de residentes) e da propriedade (como tamanho, atual uso e cobertura da terra) foram anotadas em formulário específico.

As 458 propriedades identificadas em 2003, a partir dos lotes amostrados, correspondem de fato a 1.069 lotes originais do Incra que foram agregados e/ou desagregados ao longo dos anos. As mudanças na estrutura fundiária, particularmente o processo de consolidação de terras, foi um grande complicador da tarefa de localizar donos e residentes das propriedades. A Figura 6 ilustra dois casos típicos de mudança detectados durante o trabalho de campo: no exemplo "a", uma enorme propriedade onde se produz arroz e soja foi encontrada no lugar dos lotes originais - vê-se que a propriedade em muito excede os $9 \mathrm{~km}^{2}$ da célula selecionada; no exemplo "b", no lugar 
FIGURA 6

Exemplos de mudança na estrutura fundiária observada em 2003

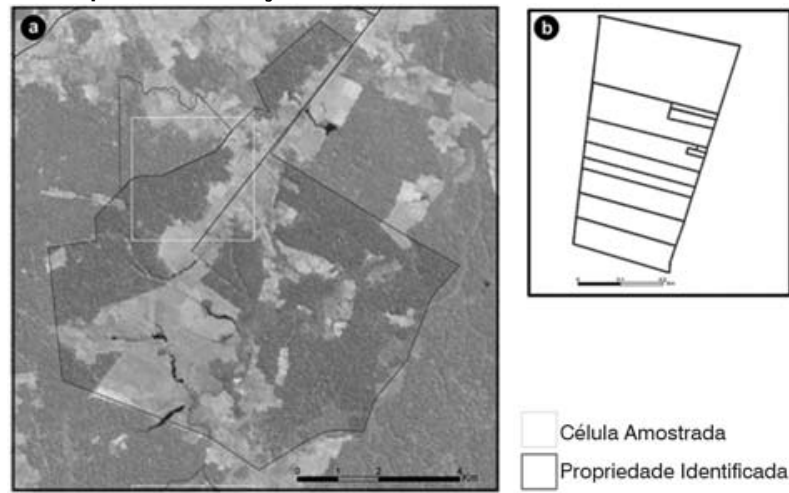

Fonte: Elaboração dos autores, a partir do acervo do ACT/Indiana University

de um lote amostrado, foram encontradas 12 propriedades ocupadas por filhos daquele que originalmente adquiriu o lote.

Os limites de todas as propriedades identificadas no campo foram atualizados segundo os desenhos feitos com os proprie-tários, os pontos de GPS coletados durante as visitas e as imagens de satélite. Do trabalho de campo até a análise dos dados, o uso de georreferenciamento permitiu estabelecer a relação entre o mapa de lotes do Incra - base da amostragem - e o mapa construído a partir das informações coletadas com as propriedades visitadas em 2003. A sobreposição dos mapas permite comparar espacialmente a estrutura fundiária do passado e a do presente. Já a sobreposição dos mapas de propriedades com as séries de imagens de satélite possibilita analisar as transformações da cobertura da terra dentro de determinado lote ou propriedade, que podem então ser associadas aos conteúdos declarados pelos entrevistados - por exemplo, a produção e as características da unidade doméstica ao longo do tempo.

\section{As vantagens da estratégia de amostragem}

\section{Relativamente a outros métodos}

Os procedimentos adotados geram uma amostra por conglomerados devido aos múltiplos estágios de seleção das unidades de amostragem (KISH, 1965). Em lugar de selecionar uma amostra aleatória de lotes ou unidades domésticas, estratificando-as por regiões na área de estudo, primeiro selecionaram-se as células com $3 \mathrm{~km} \times 3 \mathrm{~km}$ em cada região e, depois, os lotes dentro das células. Assim, os lotes são aglomerados nas células e as unidades domésticas são aglomeradas nos lotes e nas células. Tal agrupamento tem vantagens e desvantagens quando comparado à alternativa mais provável - uma amostra aleatória de lotes, com entrevistas em todas as unidades domésticas ali existentes -, conforme apresentado a seguir.

Esta amostra aleatória poderia ter sido selecionada de duas outras formas, cada qual com suas implicações. Poder-se-ia considerar uma amostra aleatória de lotes a partir da lista de lotes existentes no mapa do Incra. Esta amostra seria representativa dos lotes e unidades domésticas na região, com as unidades domésticas aglomeradas dentro dos lotes. Entretanto, a estratégia não capturaria a verdadeira variação da paisagem, porque o uso da terra e as características biofísicas dos lotes maiores seriam superdimensionados na amostra. Por exemplo, um lote com 200 hectares teria a mesma probabilidade de seleção $(1 / \mathrm{N}$, onde $N$ é o número de lotes na região) do que um outro com 4 hectares.

Outra possibilidade seria definir uma amostra aleatória de pontos e entrevistar unidades domésticas em propriedades onde tais pontos existissem, ou naquelas 
cujos centros estivessem a uma determinada distância dos pontos aleatoriamente selecionados (CRESSIE, 1993). Isto representaria a paisagem, capturando a variação em características biofísicas e no uso da terra, pois os pontos estariam dispersos ao longo de toda a área. No entanto, as propriedades não têm a mesma probabilidade de seleção, posto que as maiores delas - nas quais as unidades domésticas possuem características distintas daquelas em pequenas propriedades - têm maior chance de serem selecionadas por este procedimento. A amostra não representaria adequadamente a verdadeira variação da população de unidades domésticas, uma vez que tais unidades não estão uniformemente distribuídas ao longo da paisagem.

Nossa abordagem é um híbrido das duas possibilidades anteriormente apontadas. A seleção aleatória de unidades espaciais (células) garante a representação da variabilidade em características da paisagem, enquanto o tamanho relativamente grande das células assegura a representação da variabilidade das características das unidades domésticas. A substancial redução nos custos do levantamento de dados em propriedades aglomeradas constitui outra vantagem do procedimento adotado.

Essa estratégia de amostragem apresenta duas desvantagens que afetam a análise dos dados. Primeiramente, tendo em vista que as células foram selecionadas com igual probabilidade para garantir a representação da variação ao longo da paisagem, em lugar da seleção com probabilidade proporcional ao tamanho (número de lotes ou de unidades domésticas), a amostra não é autoponderada, sendo necessária a construção de ponderações complexas para estimar características da população de propriedades ou de unidades domésticas. Em segundo lugar, como ocorre com qualquer amostra conglomerada, unidades dentro de um grupo ou aglomerado são mais similares entre si do que com outras unidades em diferentes grupos. Deve-se ainda explorar as implicações disso nos modelos, que devem levar em conta estatisticamente a proximidade espacial (vizinhança) dos lotes. $\mathrm{O}$ aspecto positivo disso é que a estratégia adotada permite estimar os efeitos da autocorrelação espacial melhor do que em uma amostragem não segmentada.

\section{No uso dos dados coletados}

Do modo como foram coletados e organizados, os dados podem ser usados em diferentes níveis de agregação, atendendo a diversas análises. Seja na escala do estabelecimento rural, seja na da unidade doméstica, ou dos indivíduos, as variáveis podem ser analisadas temática e isoladamente (por exemplo, uso de contraceptivos entre a população feminina), ou em relação a caracteres biofísicos, tais como cobertura florestal, tipo de solo, topografia e distância de cursos d'água (por exemplo, a relação entre a composição da unidade doméstica e o tipo de cobertura florestal em sua propriedade), entre outros elementos da paisagem. Organizadas em regiões, pode-se não somente comparar efeitos do tempo de ocupação, mas também verificar as implicações das vias de acesso sobre as decisões dos produtores.

Assim, mudanças na estrutura fundiária em escala regional são analisadas a partir do uso da amostra de lotes originais do Incra $(N=587)$, podendo ser combinados à malha de propriedades identificadas em campo $(N=460)$, às imagens de satélite e aos dados do survey. As mudanças na estrutura fundiária podem ser discutidas à luz dos processos socioeconômicos e demográficos em curso (transformações tecnológicas, urbanização e migração, por exemplo), como também com relação às mudanças no uso e cobertura da terra. A localização dos casos estudados permite a comparação dos resultados com os de outras fontes, de tal modo que se particularize a área de estudo e a relacione com o município e a região.

Análises dos efeitos da demografia das unidades domésticas - tais como da composição familiar e do ciclo de vida das unidades - sobre o uso da terra na escala das propriedades familiares $(N=243)$ são generalizáveis à região. Também generalizáveis são as análises na escala das unidades domésticas (todas em propriedades familia- 
res, $\mathrm{N}=401$ ), como, por exemplo, a análise dos determinantes da co-residência de filhos adultos nas propriedades dos seus pais. Um quarto nível analítico se dá pela agregação de dados individuais, tais como a análise da escolha de métodos contraceptivos entre mulheres (com mais de 15 anos vivendo em todas as unidades domésticas em todas as propriedades familiares, $\mathrm{N}=479$ ).

\section{Nos estudos de população e ambiente}

Apresenta-se uma solução de amostragem que proporciona generalizações ao nível da paisagem - ainda que tenha sido aplicada primordialmente ao estudo do uso e da cobertura da terra - e da população de unidades domésticas. A estratégia facilita a logística de campo e pode ser adaptada a outros contextos e objetivos, através do ajuste no tamanho da célula em combinação ao número de células e à quantidade de unidades selecionadas em cada uma delas.

O entendimento das relações recíprocas entre mudanças populacionais e

\section{Referências bibliográficas}

BATISTELLA, M.; MORAN, E. F. Dimensões humanas do uso e cobertura das terras na Amazônia: uma contribuição do LBA. Acta Amazonica, v.35, n. 2, p.239-247, 2005.

BRONDÍZIO, E. et al. Land cover in the Amazon estuary: linking of the thematic mapper with botanical and historical data. Photogrammetric Engineering \& Remote Sensing, v.62, n.8, p.921-929, 1996.

BRONDÍZIO, E et al. The colonist footprint. Toward a conceptual framework of land use and deforestation trajectories among small farmers in the Amazonian frontier. In: WOOD, C. H.; PORRO, R. (Eds.). Deforestation and land use in the Amazon. University Press of Florida, 2002, p.133-161.

BROWDER, J. O. et al. Land use patterns in the brazilian Amazon: comparative farm-level evidence from Rondônia. Human Ecology: an Interdisciplinary Journal, v.32, n.2, April p.197-224(28), 2004. mudanças ambientais - seja em estudo do uso e na cobertura da terra, seja em outra análise - exige o conhecimento de ambos os tipos de processos. Para tanto, em análises micro, faz-se necessário o uso de estratégias de amostragem que permitam generalizações em termos da população de lotes e da população de unidades domésticas em determinada região. A generalização ao nível da paisagem é mais bem obtida a partir da amostra de lotes, contudo devem ser entrevistadas todas as unidades domésticas em tais lotes para garantir que a amostra seja também generalizável para a população de unidades domésticas na região. Sem tais amostras não é possível entender os efeitos, por exemplo, da titularidade da terra ou do tamanho da propriedade nas decisões dos filhos em deixar a agricultura - ou mesmo deixar a propriedade de seus pais - ou da decisão das mulheres em deixar de ter filhos. $O$ estudo de tais elementos em várias gerações são chaves para o entendimento do futuro da população e do ambiente na Amazônia.

CRESSIE, N. Statistics for spatial data. New York: J. Wiley, 1993.

EVANS, T. P.; MORAN, E. F. Spatial integration of social and biophysical factors related to landcover change. In: LUTZ, W. et al. (Eds.).

Population and environment: methods of analysis, Supplement to v. 28, 2002, p.165186.

FEARNSIDE, P. M. Human carrying capacity of the brazilian rainforest. New York: Columbia University Press, 1986.

GUILHON, N. Confederados em Santarém. Rio de Janeiro: Presença, 1983.

INSTITUTO BRASILEIRO DE GEOGRAFIA E ESTATÍSTICA - IBGE. Censo Demográfico. Brasilia: Sistema IBGE de Recuperação Automática (Sidra), IBGE, 2000.

INSTITUTO NACIONAL DE PESQUISAS ESPACIAIS - INPE. Coordenação Geral de Observação da Terra (OBT). 
Monitoramento da Floresta Amazônica por Satélite. Projeto PRODES, 2006. Disponível em: <http://www.obt.inpe.br/ prodes/>.

$\mathrm{KISH}, \mathrm{L}$. Survey sampling. New York: J. Wiley, 1965.

LIVERMAN, D.; et al. (Eds.). People and pixels: linking remote sensing and social science. Washington, D.C.: National Academy Pressed, 1998.

MARQUETTE, C. M. Land use patterns among small farmer settlers in the northeastern ecuadorian Amazon. Human Ecology: an Interdisciplinary Journal, v.26, n.4, p.573598, 1998.

MCCRACKEN, S. et al. Land use patterns on an agricultural frontier in Brazil; insights and examples from a demographic perspective. In: WOOD, C. H.; PORRO, R. (Eds.). Deforestation and land use in the Amazon. Gainsville, FL: University Press of Florida, 2002, p.162-192.

MCCRACKEN, S. D. et al. Deforestation trajectories in a frontier region of the brazilian Amazon. In: WALSH, S. J.; CREWS-MEYER, K. (Eds.). Linking people, place, and policy: a GIScience Approach. Boston: Kluwer Academic Publishers, 2002, p.215234

MCCRACKEN, S. D. et al. Remote sensing and GIS at farm property level: demography and deforestation in the brazilian Amazon. Photogrammetric Engineering \& Remote Sensing, v.65, n.11, p.1311-1320, 1999.

MORAN, E. F. Developing the Amazon. Bloomington: Indiana University Press, 1981.

MORAN, E. F. et al. Trajectories of land use: soils, succession, and crop choice. In: WOOD, C. H.; PORRO, R. (Eds.). Land use and deforestation in the Amazon. Gainsville: University of Florida Press, 2002, p.193-217.
MORAN, E. F.; BRONDIZIO, E.; VANWEY, L. Population and environment in Amazônia: landscape and household dynamics. In: ENTWISLE, B.; STERN, P. (Eds.). Population, land use, and environment. Washington, D.C.: National Academies Press, 2005, p. 106-134.

MUCHAGATA, M.; BROWN, K. Colonist farmer's perceptions of fertility and the frontier environment in eastern Amazonia. Agriculture and Human Values, v.17, n.4, p.371-384, 2000.

PICHÓN, F. J. Settler households and land-use patterns in the Amazon frontier: farm-level evidence from Ecuador. World Development, v.25, n.1, p.67-91, Jan. 1997.

REIS, A. C. F. Santarém. Rio de Janeiro: Civilização Brasileira, 1979.

SCHMINK, M.; WOOD, C. Contested frontiers in Amazonia. New York: Columbia University Press, 1992.

SIQUEIRA, A. D. et al. Women and work in a brazilian agricultural frontier. In: CLARK, G. (Ed.). Gender at work in economic life. New York: Altamira Press, 2003, p.243-267.

STEWARD, C. The Santarém agricultural landscape, Pará, Brazil: a working paper on agro-industrial and smallholder agriculture in Santarém. (Thesis in partial fulfillment of Master's of Environmental Science). Yale School of Forestry and Environmental Studies, Yale, 2004.

WALKER, R. et al. Land use and land cover change in forest frontiers: the role of household life cycles. International Regional Science Review, v.25, n.2, p.169199, 2002.

WOOD, C.; SCHMINK, M. Blaming the victim: small farmer production an Amazon Colonization Project. Studies in Third World Societies, v.7, p.77-93, 1979. 


\section{Resumen}

Estrategia para muestreo de la población y del paisaje en investigaciones sobre uso y cobertura de la tierra

Este artículo presenta la estrategia usada en una investigación realizada en Santarém - PA, en 2003, para mostrar lotes delimitados en mapas del Incra [Instituto Nacional de Colonización y Reforma Agraria] y las unidades domésticas allí existentes, de modo de captar los cambios poblacionales y ambientales en la escala de las propiedades rurales y en la de la región. La muestra fue seleccionada de acuerdo a la distribución de los lotes a lo largo de cuatro ejes viales relacionados a distintos procesos de ocupación. En cada uno de los ejes fueron seleccionadas aleatoriamente 20 células con $9 \mathrm{~km} 2$. En cada una de ellas, fueron seleccionados aleatoriamente los lotes que deberían ser visitados. En el área establecida, se entrevistaron todas las unidades domésticas existentes en cada uno de los lotes seleccionados. Se describe el muestreo por segmentación sucesiva del área de estudio, destacándose, como los datos resultantes pueden ser usados de forma diferente en las escalas de las unidades domésticas, de los lotes y de la región, de acuerdo a los aspectos y a las variables de interés.

Palabras-clave: Amazonia brasileña. Uso de la tierra. Unidad doméstica. Muestreo en períodos múltiples.

\section{Abstract}

Strategy for a sampling of the population and the land structure in studies on the use, covering and domestic units of the land

This article describes the strategy used in a study carried out in Santarém, State of Pará, Brazil, in 2003, to sample plots represented on maps produced by the Brazilian Government's Land Reform Department (INCRA). The purpose of this sampling strategy is to describe demographic and environmental changes on the scale of the rural landholdings and on a regional scale. The sample was chosen according to the distribution of plots of land along four different roads that underwent different processes of occupation. Twenty cells of $9 \mathrm{~km} 2$ each were chosen at random along each road and, in each direction, plots were chosen to be visited. In the field work, persons at all the domestic units existing in each of the plots selected were interviewed. Sampling carried out by successive segmentation of the area of study is described, as well as the way in which the resulting data can be used differently on the scales of domestic units, plots of land, and regions, according to the questions and variables of interest.

Keywords: Brazilian Amazon. Land use. Domestic unit. Sampling in multiple stages.

Recebido para publicação em 24/04/2007. Aceito para publicação em 12/11/2007. 
